\title{
Bouin's Fixative
}

National Cancer Institute

\section{Source}

National Cancer Institute. Bouin's Fixative. NCI Thesaurus. Code C63349.

A tissue fixative consisting of formaldehyde, glacial acetic acid, and picric acid. It provides particularly excellent preservation of nuclei and chromosomes. 